\title{
DESENVOLVIMENTO VISOMOTOR E USO DE JOGOS ELETRÔNICOS EM CRIANÇAS DO ENSINO FUNDAMENTAL
}

Thatiana Helena de Lima

Psicóloga e Mestranda na Universidade São Francisco - Bolsista CAPES.

\begin{abstract}
Neide de Brito Cunha
Doutora em Avaliação Psicológica Educacional da Universidade São Francisco - Bolsista CNPq de pós-doutorado.
\end{abstract}

Acácia Aparecida Angeli dos Santos

Doutora em Psicologia e docente da Universidade São Francisco - Bolsista Produtividade CNPq.

Jocemara Ferreira Mognon Psicóloga pela Universidade São Francisco, onde foi bolsista PIBIC do CNPq.

\section{Resumo}

Este estudo investiga as possíveis relações entre a maturidade percepto-motora e o uso de jogos eletrônicos, assim como possíveis diferenças entre as variáveis sexo e idade. Participaram 148 crianças do ensino fundamental, de uma instituição pública do interior do Estado de São Paulo, sendo $54,1 \%$ do sexo masculino e $45,9 \%$ do sexo feminino. Os estudantes frequentavam da $2^{a}$ a $4^{a}$ série. Foram aplicados de forma coletiva, o Teste de Bender (B-SPG) e um questionário para avaliar os padrões de uso dos jogos eletrônicos. Os resultados indicaram que os meninos utilizavam mais tempo nos jogos eletrônicos. Houve correlações positivas entre a utilização dos jogos e as pontuações no teste Bender, indicando que as crianças que mais jogavam podiam ter maiores dificuldades visomotoras. Não foram encontradas diferenças significativas para o sexo, já para a idade conforme houve avanço, menor foi a pontuação.

Palavras-chave: desenvolvimento visomotor, jogos eletrônicos, ensino fundamental.

\section{VISOMOTOR DEVELOPMENT AND USE OF ELECTRONIC GAMES IN ELEMENTARY SCHOOL CHILDREN}

\begin{abstract}
This study investigates the relationships between the perceptual-motor maturity and the use of electronic games, as well the differences between gender and age. 148 children of elementary school participated, of a public institution from Brazil, with $54.1 \%$ males and $45.9 \%$ female. Students were attending the 2nd to 4th grade. The tests were applied in a collective way, the Bender Test (B-SPG) and a questionnaire to assess the standards of use of electronic games. The results indicated that boys spent more time on electronic games. There was significant correlation between the use of games and the scores of Bender, indicating that children who played more could have more visomotor difficulties. To Bender test no significant differences were found for sex, but to age there have been advances lower was the score.

Keyword: visomotor development, electronic games, elementary school.
\end{abstract}




\title{
DESARROLLO VISOMOTOR Y USO DE JUEGOS ELECTRÓNICOS EN LA ESCUELA PRIMARIA
}

\begin{abstract}
Resumen
Este estudio investiga las relaciones entre la maduración perceptivo-motora y el uso de juegos electrónicos, así como las posibles diferencias entre el género y la edad. Participaron 148 niños que asistían a la escuela primaria, una institución pública en el estado de São Paulo-Brasil. El 54,1\% eran varones y $45,9 \%$ mujeres que del 20 al 40 grado. Se aplicaron de forma colectiva, el Bender (B-SPG) y un cuestionario para evaluar los patrones de uso de juegos electrónicos. Los resultados indicaron que los niños pasaban más tiempo en los juegos electrónicos. Hubo correlaciones positivas y significativas entre el uso de juegos y los resultados del test Bender, lo que indica que los niños que jugaban más podrían tener mayores dificultades visomotoras. No se encontraron diferencias significativas por sexo, en cuanto a la edad de acuerdo con el avance, menor fue la puntuación.
\end{abstract}

Palabras clave: desarrollo visomotor, juegos electrónicos, escuela primaria.

\section{INTRODUÇÃO}

A brincadeira é uma das melhores formas de se favorecer o desenvolvimento adequado, visto que é por meio dela que se dá a aprendizagem dos limites e a diferenciação de papéis na sociedade. Nesse sentido, o brincar propicia condições para que haja interação com outras crianças, o conhecimento de realidades diferentes, conhecimento sobre os comportamentos socialmente adequados para a sua idade e sexo e, também, a possibilidade de preparar-se para o futuro (McHale, Crouter \& Tucker, 1999).

Por meio do intercâmbio social há a ampliação da linguagem, dos conhecimentos e o reconhecimento das habilidades (Martins Filho, 2010). A brincadeira também implica na aprendizagem social, em que são aprendidas as regras e o seu momento de enunciá-las, as formas, o vocabulário característico, as habilidades específicas exigidas para cada atividade (Brougère, 2004).

Alguns autores buscam definir o brincar e sua importância para o desenvolvimento infantil. Bomtempo, Hussein e Zamberlan (1986) comentam que a brincadeira possui um fim em si mesma, envolve a busca pelo prazer e é uma atividade espontânea que proporciona à criança condições saudáveis para o desenvolvimento biopsicossocial. No mesmo sentido, Kishimoto (2007) aponta características do brincar referindo-se ao fato de a realidade interna predominar sobre a externa; que envolve ação espontânea e livre da criança; gera prazer e alegria; é composta de atividades flexíveis; tem a prioridade das crianças e é controlada internamente por seus jogadores. 
Alves (2001) considera brincadeira qualquer atividade aceita pelo simples prazer do desafio. De acordo com Piaget (1975), o brincar é importante porque possui relação com a realidade e a fantasia, visto que, quando a criança brinca, ela assimila o mundo a sua maneira, pois a interação não depende do objeto propriamente dito, mas do papel que é atribuído ao mesmo. Portanto, a brincadeira é de grande importância para a comunicação, mesmo quando a criança a faz sozinha, pois dá formas a sua imaginação.

No entanto, observa-se que, conforme aumenta a idade da criança, os brinquedos começam a dar lugar aos jogos que imitam as regras sociais dos mais velhos, com o intuito de preparar a criança para as suas responsabilidades. Por mais que haja estudos que demonstrem a importância do brincar para o desenvolvimento de uma criança, muitos adultos não veem o brincar como essencial para o desenvolvimento cognitivo da criança, embora os estudiosos afirmem que existe relação direta com a aprendizagem, sendo também fundamental para o desenvolvimento da personalidade, dos aspectos afetivo e social (Queiroz, Maciel \& Branco, 2006).

Existe uma surpreendente unanimidade de opiniões entre psicólogos, pedagogos e profissionais que cuidam da infância de uma maneira geral, que apontam para a importância das manifestações lúdicas como o modo espontâneo das crianças, de todos os grupos sociais e etnias interagirem, se expressarem e se desenvolverem. As brincadeiras podem ser vistas como acontecimentos culturais, criados e recriados em cada período e lugar e conduzidos por meio de grupos de brincadeira, geralmente multietários. É isso que caracteriza a variedade de brincadeiras, de objetos utilizados e de regras (Hansen, Macarini, Martins, Wanderlind \& Vieira 2007).

Apesar dos termos jogos e brinquedos serem utilizados de forma semelhante, eles se diferenciam da seguinte maneira, o jogo pode indicar brincadeiras que envolvem regras, enquanto que o brinquedo pode referir-se a atividades não-estruturadas e em que $\mathrm{o}$ faz-de-conta mostra-se mais evidenciado. Os jogos são uma forma de brincar que envolve a ritualização de papéis e a regulação de determinados cenários (Rosseti \& Souza, 2005).

O avanço da tecnologia trouxe novas formas de brincar, graças à propagação dos computadores que, no Brasil, deu-se nos anos 1990. Sabe-se que, no final de 2000, eram 11 milhões os computadores instalados e 10 milhões de usuários conectados à internet. Apesar de a tecnologia estar disponível a 
apenas uma parcela pequena da população, é grande o número de crianças e adolescentes que se apropriam das novas tecnologias (Hoff \& Wechsler, 2002).

Nessa direção, outra forma de diversão hoje apresentada são os jogos eletrônicos, que são aqueles utilizados no computador e nos videogames. Dessa forma, juntamente aos computadores houve outro fenômeno típico da mesma época, sendo ele a popularização em massa dos jogos eletrônicos entre crianças e adolescentes. Esse fenômeno tem despertado grande interesse entre os pesquisadores e colocado os jogos eletrônicos entre os mais utilizados pela população jovem do país (Rosseti \& Souza, 2005). Algumas das pesquisas recuperadas nas bases de dados são apresentadas a seguir.

Segundo Fortim (2006), os jogos eletrônicos podem ser de diferentes formas. São capazes de proporcionar não só a interação entre homem e máquina, mas também a interação dos homens por meio da máquina. Os jogos eletrônicos mais conhecidos são os que simulam realidades virtuais. Acrescenta, ainda, que há um fascínio dos jovens pelo mundo dos jogos eletrônicos, porque eles representam a cultura lúdica infantil. No entanto, a autora se refere ao fato de que há pesquisadores que defendem e outros que se contrapõem aos jogos eletrônicos. Isso ocorre porque ainda não há acúmulo de conhecimento sobre seus efeitos no comportamento de quem os utiliza. Exemplo dessa discussão já era visível nas considerações de Tapscott (1999), ao referir-se à utilização que as crianças fazem da tecnologia, quando coloca que as crianças (e jovens) usam - computador para atividades que caminham lado a lado com nossa compreensão do que seja uma infância tradicional. Elas usam a tecnologia para brincar, aprender, comunicar-se e formar relacionamentos, como sempre fizeram. Porém, a mídia digital está criando um ambiente no qual essas atividades próprias da infância estão mudando radicalmente e poderão acelerar, para o melhor e o pior, o desenvolvimento infantil.

Rossetti, Smarssaro e Pessotti (2009) realizaram um trabalho com o propósito de investigar o panorama atual da prática de jogos e brincadeiras em diferentes municípios do Espírito Santo. Realizaram 205 observações de atividades lúdicas de crianças de sete a doze anos, sendo 105 do interior e 100 da capital. Algumas crianças responderam a uma entrevista sobre preferências e práticas lúdicas. Pôde-se perceber nos resultados que tanto no interior quanto na capital a brincadeira mais citada foi o futebol em suas diferentes modalidades. Entre os meninos a brincadeira preferida é o futebol $(66,7 \%)$, já entre as 
meninas é a com boneca (25\%). Jogos no computador foram um dos citados entre os jogos praticados, diferentes do preferido. Segundo os autores, o estudo contribui para desmistificar a ideia de que as crianças não praticam mais brincadeiras tradicionais.

O estudo de Cordazzo e Vieira (2008) objetivou investigar os tipos de brincadeiras utilizadas por crianças do ensino fundamental. Para tanto, utilizaram uma entrevista com as crianças em sala de aula e observação nos recreios. Dentre os resultados, detectaram que algumas brincadeiras são comuns a todas as idades, como as que exigem coordenação motora ampla, dentre elas os jogos eletrônicos e os de faz de conta. Observaram que a diversidade de brincadeiras e a quantidade de eventos diminuem entre as crianças mais velhas, principalmente entre as meninas.

Observa-se que o desenvolvimento psicomotor está relacionado com o brincar. Buriti Neto (2001) descreve a relação das brincadeiras com a estruturação das condutas motoras de base, neuromotoras e as perceptivomotoras. Assim, a utilização de jogos eletrônicos desenvolve as condutas relacionadas à coordenação viso-manual, organização espacial e estruturação espaço-temporal. Ruiz (1992, citado por Rosseti \& Souza, 2005) aponta três subcategorias de jogos dentro dos jogos de ação. Primeiro estão os que resultam dos comportamentos percepto-motores, que são os motores-manipulativos. Em seguida, estão os jogos de sucesso, que dão prazer e têm a finalidade de construir ou conseguir algo. Por fim, estão os jogos eletrônicos, que possuem um forte componente de ação.

Há discussões sobre os benefícios e malefícios que os jogos eletrônicos proporcionam nas crianças. Andrade e cols. (2003) apontam que os jogos eletrônicos usados com objetivos definidos podem desenvolver várias capacidades cognitivas. Além do domínio cognitivo, o domínio psicomotor também é contemplado nos jogos eletrônicos. A percepção visual e a coordenação motora são fundamentais para o jogador interagir com o ambiente do jogo.

Durkin e Barber (2002) salientam que os jogos eletrônicos podem proporcionar habilidades mecânicas, maior capacidade de liderança, inteligência mais elevada, melhor autoconceito, maior habilidade para computador, níveis mais altos de proximidade familiar e de amizades menos arriscadas do que os que não utilizam os jogos. No entanto, algumas pesquisas têm alertado quanto 
ao fato de as crianças que não praticam nenhum tipo de brincadeira ativa e que passam muitas horas em frente à televisão, ou jogam por muito tempo videogame, poderem apresentar taxas metabólicas alteradas, o que pode, também, fazer com que a imaginação e a criatividade não se ampliem (Howes \& Matheson, 1992).

Oliveira (2009) aponta que a preocupação sobre os jogos eletrônicos, para muitas pessoas, é que eles são simplesmente sensório-motores, de coordenação visomotora e que, portanto não desenvolveriam outras habilidades cognitivas. Ao contrário, Alves (2001) ressalta que as funções cognitivas são intensificadas com os jogos eletrônicos e permitem aos seus usuários a descoberta de novas formas de conhecimento, que ocorrem na interação com os jogos. Já Greenfield (1988) ressalta que o desenvolvimento das habilidades sensório-motoras, como a coordenação visomotora, é importante por si só, sendo fundamental para o jogador interagir com o jogo.

Preocupada com formas de avaliar a habilidade visomotora, Lauretta Bender (1955) adaptou as figuras originais de gestalt visual usadas por Wertheimer e transformou-as em uma tarefa visomotora, solicitando às crianças que copiassem os modelos apresentados. A autora demonstrou a relação da atividade de cópia de figuras gestálticas e o desenvolvimento visomotor (Suehiro \& Santos, 2006). Muitos sistemas de correção foram desenvolvidos posteriormente, usando as mesmas figuras criadas por Bender para aferir quantitativamente o nível de maturidade de crianças saudáveis ou com problemas de atraso no desenvolvimento e de aprendizagem, entre outros.

A habilidade visomotora tem sido desde então mensurada pelo teste de Bender, bastante utilizado no Brasil. O sistema de avaliação do Bender, proposto por Koppitz (1989), pressupõe que essa habilidade está associada a outras funções cognitivas, tais como percepção visual, habilidade motora manual, conceitos temporais e espaciais e organização ou representação. Ademais, a percepção e a reprodução das figuras gestálticas de Bender são influenciadas por princípios biológicos e de ação sensório-motora, que variam de indivíduo para indivíduo, em razão do padrão de desenvolvimento de cada um, bem como de eventuais alterações patológicas funcionais ou orgânicas.

Embora muito utilizado, o sistema Koppitz recebeu várias críticas de estudiosos estrangeiros e brasileiros. Entre as pesquisas que apontaram seus principais problemas, destacam-se as de Bartholomeu, Rueda e Sisto (2005); 
Sisto, Noronha e Santos (2004); Suehiro e Santos (2005) que fizeram sérios questionamentos sobre esse sistema de avaliação, apontando para a necessidade de uma ampla revisão. Essa foi a principal justificativa para o desenvolvimento do Teste Gestáltico Visomotor de Bender - Sistema de Pontuação Gradual, publicado recentemente (B-SPG) por Sisto, Noronha e Santos (2006), tendo como embasamento os pressupostos teóricos de Bender (1955).

Em estudo realizado por Noronha, Santos e Sisto (2007), o objetivo foi buscar evidências de validade para o teste de Bender - Sistema de Pontuação Gradual. Participaram 1052 crianças de ambos os sexos, da pré-escola e do ensino fundamental. Os achados mostraram que conforme a idade das crianças aumenta, diminui a pontuação no Bender. Quanto aos sexos, não foram encontradas diferenças significativas.

Nesse sentido, identificaram-se na literatura apenas estudos que têm demonstrado que o desenvolvimento psicomotor está associado a atividades que as crianças praticam em suas brincadeiras (Buturi Neto, 2001; Fortim, 2006). Não foram encontradas pesquisas que relacionem o desenvolvimento visomotor com a utilização de jogos. Assim, considerando o uso cada vez mais frequente dos jogos eletrônicos, julgou-se relevante investigar as possíveis relações entre o seu uso e o desenvolvimento visomotor de alunos do ensino fundamental. $O$ presente estudo tem como objetivo analisar possíveis relações entre o uso de jogos eletrônicos e o desenvolvimento visomotor dos estudantes, assim como possíveis diferenças entre as variáveis sexo e idade.

\section{MÉTODO}

\section{Participantes}

Participaram do estudo 148 crianças do ensino fundamental, provenientes de uma instituição pública do interior do Estado de São Paulo, sendo 54,1\% $(N=80)$ do sexo masculino e $45,9 \%(N=68)$ do sexo feminino. Desses, 52 $(35,1 \%)$ eram da segunda série, $45(30,4 \%)$ da terceira e $51(34,5 \%)$ da quarta, com idade variando de 7 a 13 anos $(M=9,2 ; D P=1,25)$.

\section{Instrumentos}

Teste Gestáltico Visomotor de Bender - Sistema de pontuação gradual (B-SPG)

Desenvolvido por Sisto, Noronha e Santos (2006), o Sistema de Pontuação Gradual (B-SPG) avalia a maturidade visomotora com base nos pressupostos 
teóricos preconizados por Bender, que propõe que, a partir da reprodução de desenhos, é possível identificar o nível de maturação da função gestáltica visomotora. Consiste de nove figuras $(A, 1,2,3,4,5,6,7$ e 8) para serem copiadas da melhor maneira possível, sem a permissão para utilização de borracha ou qualquer outro tipo de material que sirva como suporte mecânico. 0 teste destina-se a crianças de 6 a 10 anos e pode ser aplicado de forma individual ou coletivamente por meio de transparências. A correção do instrumento implica na atribuição de uma nota de 0 até 2 pontos, sendo que somente no caso da Figura 6 a pontuação pode variar de 0 a 3 pontos, para os erros cometidos na reprodução gráfica dos modelos de Bender, o que varia em razão da qualidade da cópia realizada, conforme orientação do manual. O total de pontos possíveis, referentes aos erros de distorção de forma cometidos, é de 21 pontos.

Questionário sobre Padrões de Uso de Jogos Eletrônicos (Barbosa, 2008)

Constituído por 10 questões, das quais 7 são fechadas e 3 abertas, sendo que algumas delas têm mais que uma alternativa que pode ser assinalada. Dessa forma, identifica-se a caracterização do participante; a frequência que utiliza os jogos eletrônicos (videogame, jogos de computador, no celular ou minigames), possibilitando ao participante escolher mais de uma alternativa; os motivos pessoais que levam o participante a jogar; as preferências de jogos; a duração de horas por dia; a história de uso de jogos eletrônicos e se o participante tem interesse por jogos que não são eletrônicos, como tabuleiros, baralhos e jogos esportivos (futebol, vôlei e outros). Foi atribuído um ponto para cada alternativa assinalada pela criança.

\section{Procedimento}

Após a aprovação do projeto pelo Comitê de Ética e a assinatura do Termo de Consentimento Livre e Esclarecido pelos pais das crianças, foram agendados com a escola datas e horários para realizar a aplicação. A coleta de dados foi feita coletivamente com supervisão de, pelo menos, dois pesquisadores e os instrumentos foram aplicados um de cada vez, obedecendo a seguinte ordem: Bender - Sistema de Pontuação Gradual (B-SPG) e o Questionário sobre Padrões de Uso de Jogos Eletrônicos. A aplicação ocorreu em 40 minutos. 


\section{RESULTADOS}

Serão apresentadas, primeiramente, correlações alcançadas entre o Bender - Sistema de Pontuação Gradual e o Questionário sobre Padrões de Uso de Jogos de Jogos Eletrônicos e após as diferenças entre sexo e idade para cada um deles. A fim de averiguar se havia correlação entre ambos os instrumentos, utilizou-se a Correlação de Pearson (nível de significância de 0,05).

Observou-se correlação positiva e significativa entre a pontuação obtida no Bender e a quantidade de horas que os sujeitos utilizam jogos eletrônicos $(r=0,21 ; p=0,024)$, o que revela que as crianças com maior pontuação no Bender passam mais tempo jogando, assim quanto menor o desenvolvimento visomotor, maior a quantidade de horas que os indivíduos jogam. Quanto às diferenças encontradas no Questionário de Jogos Eletrônicos para o sexo na Tabela 1 pode-se observar alguns dos resultados obtidos.

\section{Tabela 1.}

Médias, desvio-padrão, valores de t e $p$ em relação ao Questionário de Jogos Eletrônicos, por quantidade de dias utilizados, variedade e horas de uso.

\begin{tabular}{ccccccc}
\hline & Sexo & $\mathrm{N}$ & Média & $\begin{array}{c}\text { Desvio } \\
\text { Padrão }\end{array}$ & $\mathrm{t}$ & $p$ \\
\hline Dias & Masculino & 76 & 5,68 & 2,811 & 3,498 & 0,001 \\
& Feminino & 65 & 4,03 & 2,784 & & \\
Variedade & Masculino & 80 & 2,41 & 1,338 & 1,237 & 0,218 \\
& Feminino & 68 & 2,16 & 1,087 & & \\
Horas de & Masculino & 77 & 5,03 & 7,574 & 2,795 & 0,006 \\
uso & Feminino & 67 & 2,31 & 2,554 & & \\
& & & & & &
\end{tabular}

O sexo masculino obteve maior escore nos três aspectos avaliados, ou seja, na quantidade de dias, na variedade de suporte (computador, vídeo e "minigames", celular etc.) e na quantidade de horas disponibilizadas para a utilização dos jogos eletrônicos. No entanto, a diferença significativa entre os sexos apresentou-se somente na quantidade de dias e horas utilizadas para jogar. Quanto à variável idade, não foram observadas diferenças significativas.

Na pontuação média do Bender, quando feita a comparação entre os sexos, não houve diferença significativa $(t=0,708 ; p=0,480)$, esta foi apresentada quando considerada a variável idade. Com a aplicação da $\operatorname{ANOVA}[F(3,120)=$ $11,402 ; p<0,001]$ e por meio da prova ad-hoc de Tukey os grupos foram separados, tal como apresentado na Tabela 2. 
Tabela 2.

Distribuição das pontuações obtidas no Bender em grupos por idade.

\begin{tabular}{ccccc}
\hline Idade & $\mathrm{N}$ & \multicolumn{3}{c}{ Pontuação do Bender } \\
\cline { 3 - 5 } & & 1 & 2 & 3 \\
\hline 10 & 43 & 7,09 & & \\
9 & 38 & 9,55 & 9,55 & \\
8 & 31 & & 11,19 & 11,19 \\
7 & 12 & & & 12,42 \\
$p$ & & 0,084 & 0,386 & 0,635 \\
\hline
\end{tabular}

Por meio desses dados, verificou-se que a pontuação obtida pelos sujeitos no Bender decresce conforme aumenta a idade. Cabe lembrar que o Bender pontua o erro, ou seja, quanto maior o número de erros que o indivíduo alcançar maior será a sua pontuação. Assim, espera-se que, quanto maior a idade, maior o desenvolvimento visomotor da criança e, portanto, menor o número de erros o que, consequentemente, acarreta uma menor pontuação.

\section{DISCUSSÃO}

Diante dos resultados apresentados anteriormente, pode-se inferir que as crianças que participaram do estudo obtiveram médias ditas como esperadas para ambos os instrumentos. Assim sendo, no teste de Bender (B-SPG), quanto maior a idade menor a pontuação. O resultado encontrado corrobora as pesquisas de Noronha, Santos e Sisto (2007) e Suehiro e Santos (2006), visto que ao se comparar o sexo e no teste de Bender não foram encontradas diferenças significativas. Isso está em consonância com outras pesquisas que têm ressaltado que não há evidências de que meninos e meninas se diferenciem no processo de amadurecimento percepto-motor como a de Noronha, Santos e Sisto (2007).

Observou-se que quanto maior a idade do sujeito mais tempo ele disponibiliza para a utilização de jogos eletrônicos. Rosseti \& Souza (2005) já haviam observado que esses jogos estão entre os mais utilizados pela população jovem do país. Esses fatos corroboram o estudo de Cordazzo e Vieira (2008), que encontrou que a diversidade de brincadeiras e a frequência diminuem entre as crianças mais velhas. Cabe ressaltar que, para Fortim (2006), os jogos eletrônicos mais conhecidos são os que simulam realidades virtuais. A autora 
acrescenta que há um fascínio dos jovens pelo mundo dos jogos eletrônicos, porque eles representam a cultura lúdica infantil.

Quando comparados os escores obtidos, houve correlação positiva e significativa, com magnitude baixa, indicando que, quanto mais os indivíduos jogam menor a sua habilidade visomotora. Esse resultado vem ao encontro da opinião de Oliveira (2009) que acredita que os jogos eletrônicos não desenvolvem habilidades cognitivas. Entretanto, vai de encontro ao estudo de Alves (2001), que mostra que os jogos intensificam as funções cognitivas porque permitem a descoberta de novas formas de conhecimento. O resultado também se opõe ao de Andrade e cols. (2003), os quais apontaram que os jogos eletrônicos abrangem muito mais que os domínios cognitivos e psicomotores, como a percepção visual e a coordenação motora, que são fundamentais para o indivíduo interagir com o ambiente do jogo. Greenfield (1988) complementa que o desenvolvimento das habilidades sensório-motoras, como a coordenação visomotora, é importante por si só, sendo fundamental para o jogador interagir com o jogo.

Diante das discussões apresentadas e da preocupação dos pesquisadores quanto à utilização dos jogos eletrônicos, quanto a seus benefícios e malefícios, vale lembrar que o trabalho de Rossetti, Smarssaro e Pessotti (2009) contribuiu para desmistificar a ideia de que as crianças não praticam mais brincadeiras tradicionais, pois os resultados apontaram que tanto no interior quanto na capital a brincadeira mais citada foi o futebol em suas diferentes modalidades.

\section{CONSIDERAÇÕES FINAIS}

Devido ao fato do crescimento da tecnologia e dos jogos eletrônicos nos últimos tempos, julga-se necessário que haja mais estudos que demonstrem seus efeitos no desenvolvimento de crianças. Percebe-se que, cada vez mais, meninas e meninos têm utilizado aparelhos com a chamada "tecnologia de ponta" e muitas vezes há um excesso nessa utilização, podendo comprometer aspectos do desenvolvimento infantil.

Acredita-se, no entanto, que os jogos eletrônicos, provenientes dessa tecnologia, podem e devem ser utilizados, mas o seu uso deve ser moderado. Sabe-se o papel importante que eles podem ter para a educação, quando guiados por objetivos psicopedagógicos bem definidos, pois podem desenvolver várias capacidades cognitivas. Além disso, os jogos eletrônicos na escola podem 
possibilitar o desenvolvimento nos alunos dos aspectos sociais, afetivos e culturais.

Os jogos eletrônicos, por sua grande utilização, instigam a curiosidade e a preocupação dos pais e dos educadores em relação aos efeitos na cognição e nos aspectos motores da criança. Esses questionamentos podem ser sanados com pesquisas nessa área que podem ajudar desmistificar ou até reforçar outros achados.

Nesta pesquisa, algumas limitações podem ser apresentadas, como o questionário de jogos eletrônicos, que pode ser aperfeiçoado para avaliar o que se pretende. Por conta da faixa etária, as crianças que participaram da pesquisa podem não ter conseguido estimar, adequadamente, o número de horas que utilizavam os jogos eletrônicos. Dessa forma, sugere-se, que sejam realizados outros estudos para obtenção de maiores informações em relação também a outros aspectos cognitivos.

\section{REFERÊNCIAS}

Alves, R. (2001). É brincando que se aprende. Páginas Abertas, 27, 20-21. Andrade, L., Zavaleta, J., Vaz, F., Lima, C., Araujo, C., \& Soares, A. (2003). Jogos Inteligentes são Educacionais? Em $14^{\circ}$ Simpósio Brasileiro de Informática na Educação, NCE, IM/UFJF. Disponível em www.nce.ufrj.br/sbie2003/publicacoes/.pdf. Recuperado em 30 nov. 2010.

Barbosa, A. J. G. (2008). Questionário sobre padrões de uso de jogos eletrônicos. Relatório técnico. Juiz de Fora: Universidade Federal de Juiz de Fora.

Bartholomeu, D., Rueda, F. J. M., \& Sisto, F. F. (2005). Teste de Bender e dificuldades de aprendizagem: quão válido é o sistema Koppitz? Avaliação Psicológica, 5(1), 13-22.

Bender, L. (1955). Test Gestáltico Visomotor. Buenos Aires: Editorial Paidós. Bomtempo, E., Hussein, L. H., \& Zamberlan, M. A. T. (1986). Psicologia do brinquedo: Aspectos teóricos e metodológicos. São Paulo: Editora da Universidade de São Paulo.

Brougère, G. (2004). Brinquedo e cultura. São Paulo: Cortez.

Buturi Neto, S. T. (2001). A importância do brincar para o desenvolvimento infantil. Monografia de Especialização, Pontifícia Universidade Católica do Paraná, Curitiba/PR. 
Cordazzo, S. T. D., \& Vieira, M. L. (2008). Caracterização de brincadeiras de crianças em idade escolar. Psicologia: Reflexão e Crítica, 21(3), 365-373.

Durkin, K., \& Barber, B. (2002). Not so doomed: Computer game play and positive adolescent development. Journal of Applied Developmental Psychology, 23, 373-392.

Fortim, I. (2006). Alice no país do espelho: o MUD - o jogo e a realidade virtual baseados em textos. Imaginários, 12(12), 171-194.

Greenfield, P. M. (1988). O Desenvolvimento do Raciocínio na Era da Eletrônica. São Paulo: Summus.

Hansen, J., Macarini, S. M., Martins, G. D. F., Wanderlind, F. H., \& Vieira, M. L. (2007). O brincar e suas implicações para o desenvolvimento infantil a partir da psicologia evolucionista. Revista Brasileira de Crescimento e Desenvolvimento Humano, 17(2), 133-143.

Hoff, M. S., \& Wechsler, S. M. (2002). A prática de jogos computadorizados em um grupo de adolescentes. Estudos de Psicologia, 19(2), 59-77.

Howes, C., \& Matheson, C. C. (1992). Sequences in the development of competent play with peers: social and social pretend play. Developmental Psychology, 28(5), 961-974.

Kishimoto, T. M. (2007). Jogo, brinquedo, brincadeira e a educação. 5. ed. São Paulo: Cortez.

Koppitz, E. M. (1989). O Teste Gestáltico Bender para crianças. Porto Alegre: Artes Médicas.

Martins Filho, A. J. (2010). Olhares investigativos sobre as crianças: o brincar e a produção das culturas infantis. Momento, 19(1), 89-104.

McHale, S. M., Crouter, A. C., \& Tucker, C. J. (1999). Family context and gender role socialization in middle childhood: Comparing girls to boys and sisters to brothers. Child Development, 70, 990-1004.

Noronha, A. P. P., Santos, A. A. A., \& Sisto, F. F. (2007). Evidências de validade do Bender - Sistema de Pontuação Gradual (B-SPG). Psicologia: Reflexão e Crítica, 20(2), 335-341.

Oliveira, R. L. (2009). O adolescente e os jogos eletrônicos: padrões de uso e motivos. Monografia de Pós Graduação em Psicologia e Desenvolvimento Humano, Universidade Federal de Juiz de Fora, Minas Gerais.

Piaget, J. (1975). O nascimento da inteligência na criança. Rio de Janeiro, Zahar. 
Queiroz, N. L. N., Maciel, D. A., \& Branco, A. U. (2006). Brincadeira e desenvolvimento infantil: um olhar sociocultural construtivista. Paidéia, 16(34), 169-179.

Rossetti, C. B., Smarssaro, T. R., \& Pessotti, T. L. (2009). Inventário das brincadeiras e jogos de crianças em diferentes municípios do Estado do Espírito Santo. Revista de Psicopedagogia, 26(81), 388-395.

Rossetti, C. B., \& Souza, M. T. C. C. (2005). Preferência lúdica de uma amostra de crianças e adolescentes da cidade de Vitória. Psicologia: Teoria e Prática, $7(2), 87-114$.

Sisto, F. F., Noronha, A. P. P., \& Santos, A. A. A. (2004). Distorção de forma no Teste de Bender: Questionando seu critério de validade. Revista do Departamento de Psicologia da UFF, 16(2), 139-154.

Sisto, F. F., Noronha, A. P. P., \& Santos, A. A. A. (2006). Teste Gestáltico Visomotor de Bender - Sistema de Pontuação Gradual (B-SPG). São Paulo: Vetor Editora.

Suehiro, A. C. B. \& Santos, A. A. A. (2005). O Bender e as dificuldades de aprendizagem: estudo de validade de critério. Avaliação Psicológica, 4(1), 23-31.

Suehiro, A. C. B., \& Santos, A. A. A. (2006). Evidência de validade de critério do Bender-Sistema de Pontuação Gradual. Interação em Psicologia, 10(2), 217-224.

Tapscott, D. (1999). Geração Digital - A crescente e irreversível ascensão da Geração Net. São Paulo: Makron Books.

Contato: acacia.angeli@gmail.com

Recebido em: 14/10/2010

Revisado em: 17/11/2010

Aceito em: 02/12/2010 